\title{
A Problem Analysis of RSOA-Based Optical Access
}

\author{
Paulius Tervydis ${ }^{1}$, Ruta Jankuniene ${ }^{1}$ \\ ${ }^{1}$ Department of Telecommunications, Kaunas University of Technology, \\ Studentu St. 50-423, LT-51368 Kaunas, Lithuania \\ paulius.tervydis@ktu.lt
}

\begin{abstract}
Using WDM multiplexing is one of the ways to increase PON bandwidth. But as WDM-PON is still expensive and difficult administered, the idea of using a Reflective Semiconductor Optical Amplifier (RSOA) is proposed. It can be installed in WDM-PON ONU network devices. A comprehensive problem analysis of various investigations is done relating to a performance of RSOA-based WDM-PON systems. The simulation model is created for RSOA-based WDM-PON access with downstream signal reusing in ONU for upstream transmission. This model helped in researching of RSOA abilities using different downstream and upstream signals modulation. Signal parameters were tested working with special software OptiSystem. Results were evaluated regarding such a characteristics as $Q$ factor, signal attenuation, power and optical fiber length. It was found that $\mathrm{RZ}$ code was better suited in remodulation with RSOA; it achieved 1.25 Gbps signal transmission at a distance 8 times larger than that of the in NRZ code case.
\end{abstract}

Index Terms-Reflective Semiconductor Optical Amplifier (RSOA); RSOA-based WDM-PON access performance; wavelength remodulation; coding technique.

\section{INTRODUCTION}

The necessity of contemporary optical access networks reconfiguration for their ability to meet the new requirements of bandwidth $(\mathrm{BW})$ is determined by exponential growth of Internet traffic and new broadband services as well as the growing number of optical access users, while transmission distances are getting longer too. A number of investigations have been carried out for creation of new optical access network architectures and their optical components [1]. A wavelength division multiplexing (WDM) technology for optical access is considered as a promising solution because of its unlimited bandwidth, security and protocol clearness [2]. WDM technology is saturated already; however, its usage in passive optical networks (PON) is quite complicated due to the high implementation costs and compatibility problems with network technologies already installed.

A WDM-PON implementation costs directly depend on the number of network users. A high number of PON users and big transmission rates require a high capacity of ONU work modules. The usage of passive components in network part between OLT and ONU reduces overall system costs. For these reasons, low-cost modules with limited working capacity can be used which enable to implement costeffective methods, for example, offset filtering, electronic equalization or advanced forward error correction (FEC)) [3].

Another solution for WDM-PON cost reduction can be the considering of various colourless light source technologies: the splitting of the spectrum, amplified spontaneous emission (ASE) injected Fabry-Perot lasers and Reflective Semiconductor Optical Amplifier (RSOA) [4]. Those technologies assure independent functioning of optical network unit (ONU) regarding light wavelength and avoiding expensive external modulators and receivers in the ONU [2].

RSOA functioning is similar to conventional semiconductor optical amplifier (SOA) [5], however, optical gain, noise characteristics and gain saturation may be very different due to SOA (the studies of theoretical RSOA pulse propagation dynamics are still ongoing [5]). One port semiconductor device RSOA may be very effective regarding costs in case of mass production. Moreover, it ensures colour-free ONU functioning while simplifying requirements for network equipment, because there is no need to use a particular wavelength equipment and wavelength control. RSOA is an attractive choice for inexpensive ONU transmitters [3], [4], [6]-[9].

\section{RSOA BASED WDM-Pon ACCESS ARCHITECTURES}

RSOA transmitters are wide wavelength spectrum equipment and they are used in various configurations of WDM-PON (Fig. 1), i.e. for EDFA spectrum distribution (in central office (CO) or OLT), as wide spectrum laser, RSOA ASE noise use and wavelength re-use (when implementing ONU) [10].

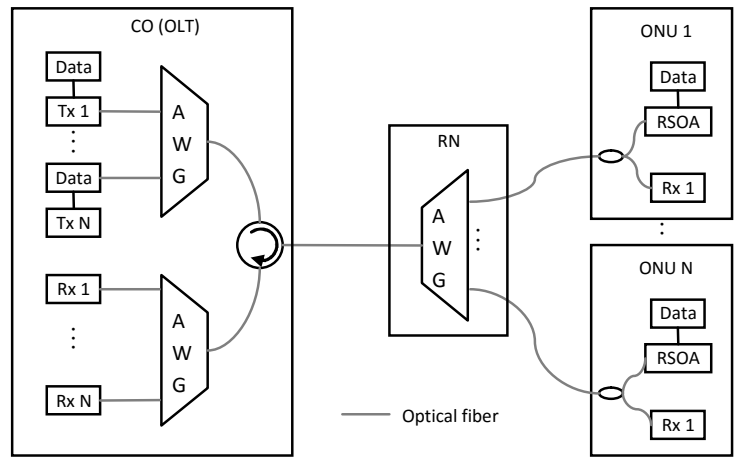

Fig. 1. RSOA-based WDM PON. 
A downstream signal and continuous wave (CW) are supplied from optical line terminal (OLT) and they are distributed through AWG and redirected to certain ONU with RSOA installed. The latter one enhances and modulates CW signal with user data and sends it back to OLT through the same fiber already as an upstream signal.

The most optimal solution regarding costs is downstream wavelength reusing for upstream signal, as there is no need for additional light source $(\mathrm{CW})$. In this case, a downstream signal is generated by directly modulated single-mode lasers (SMLs) in central office (CO) and is transmitted to optical network terminal (ONT) by means of wavelength multiplexer distributed in remote node (RN). $75 \%$ of optical power received in ONU is directed to RSOA and $25 \%$ of it is directed to optical coupler receiver. A coupling ratio is carefully determined taking into account the following: a power level of downstream signal (it must exceed the receiver sensitivity level and be within RSOA power saturation limits); the ratio of upstream signal power (because Relay scattering) and backscattering noise. The light fed to RSOA should be sufficient for the above conditions to be fulfilled. Various modulation formats were suggested to be used to solve this problem, i.e. Manchester coding and sub-carrier multiplexing (SCM) for reducing modulation noise. Thus, it is possible to use high extinction ratio of logical 1 and 0 for downstream signal and to decrease power penalties due to remodulation.

\section{RSOA BASED WDM-PON PERFORMANCE PROBLEMATICS}

The problems related to performance of RSOA based WDM-PON system (later in this document the term "system" is used) includes many factors that affect system performance, i.e. results in optical power penalty. Reflections caused by various in-band crosstalks, resulting in optical signal power penalties are especially important. In-band crosstalks, nonlinearity and chirp effects as well as optical filtering problems at high channel density are highly relevant to system performance. Due to the development of current 100 Gigabit Ethernet $(100 \mathrm{GbE})$ standards, it is extremely important to ensure high rates in optical access. All these topics are analysed in various research works.

\section{A. Influence of Reflections}

System advantages, i.e. its integrity and wavelength independence, may be lost due to the high sensitivity to dissipated or reflected light. Since the upstream waves are transmitted through the same fiber loopback as downstream signal, the performance of such systems may be vulnerable to reflections caused by fiber connections, fusions and other points in line as well as the Relay backscattering effect. Power instabilities occur due to reflections. This leads to crosstalks which degrade signal-to-noise ratio (SNR). Eventually, it may result in more bits transmission errors of upstream signal [1], [4]. The influence of various reflecting points to downstream and upstream signals was evaluated by reflection tolerance depending on RSOA gain and reflection type [4], [8]. Only a very small number of them are acceptable especially if they occur near the ONU or CO. The results of the investigation have revealed that namely the reflection of upstream signal into ONU may have the biggest influence on upstream and downstream signals and it may harm them due to its re-enhancing in RSOA. Therefore, a noise of optical beat interference (OBI) may occur. The latter was evaluated as a function of RSOA gaining, line attenuation and location of reflection [8].

A reflection tolerance may be increased by optimising RSOA gain and/or coupler splitting ratio in ONU. For practical system installation it may be necessary to use reflections reduction techniques, such as spectrum widening.

A fluctuation of amplitude is less when RSOA is in saturation condition. This requires a higher optical signal power supplied to the RSOA and it became less effective because of increased budget loss. For this reason, an influence of reflections to performance of gain-saturated RSOA was experimentally investigated in such channel architecture where a downstream signal was remodulated with upstream data [1], [4].

\section{B. Resistance to In-band Crosstalks}

The using of broadband light source (BLS) may be an effective solution for costs reduction. Unfortunately, the performance of such system degrades because a chromatic dispersion occurring in high transmission rates. In order to improve the system dispersion tolerance, it was proposed to use a distributed feedback laser diode (DFB-LD) [9]. The influence of light source type on in-band crosstalk penalty was investigated [11]. A power reduction was determined due to in-band incoherent crosstalk with two different light sources: broadband light source (BLS) and distributed feedback laser diode (DFB-LD). Optical light sources with direct modulation (irrespectively on source type) were more tolerable for in-band crosstalk penalty compared with an externally modulated DFB-LD source [11].

The influence of coherent and incoherent light sources (distributed feedback (DFB) lasers or spectrally sliced broadband source) on receiver power penalties was investigated in a number of studies [1]. Some solutions were found to decrease the in-band crosstalk penalty: an additional phase modulation, polarization coding, lowcoherent source installation and the time gap between the desired and the interfering signals. The in-band crosstalks were investigated as a sum of two phenomena due to the partly reflected or scattered signals transmitted simultaneously. RSOA bias dithering was proposed as a new method for using of amplitude-to-phase modulation in an active RSOA material [12], [13] and phase modulation in laser [14]. The in-band crosstalks were evaluated between $\mathrm{cW}$ from coherent source and data stream retransmitted from RSOA [1], [13]. It was found that RSOA was highly vulnerable to back reflections. RSOA tolerance to reflections and scattering can be significantly improved by proper phase modulation. A method for crosstalks reduction was presented applying one tone phase modulation by using RSOA nonlinearity or external phase modulator on the source side.

\section{Influence of Nonlinearities}

The RSOA is highly cost-efficient in systems with orthogonal frequency division multiplexing (OFDM). However, high to average ratio of OFDM signal power renders RSOA sensitive to linearity or distortions as the signal peaks may occasionally thrust into the saturation 
region of the RSOA. Distortions caused by RSOA are decisive factors in limiting the maximum achievable system performance. An analytical method was created to evaluate the influence of bandwidth nonlinearities on system performance [15]. RSOA gain characteristics were described numerically on the basis of nonlinear curve fitting approach. Expressions are derived for in-band and adjacent-band harmonic distortion caused by the noise as well as the optimal input power to obtain the maximum achievable transmission performance. The simulation results showed that the nonlinear effects caused the deterioration of transmission and they are highly dependent on the third order term of nonlinear distortion power.

\section{Neutralization of Chirp Factor}

RSOA has a frequency chirp which results in optical signal power fading. RSOA chirp is one of the most important limiting factors in systems with OFDM and not only does it reduce transmission distance but the maximum capacity as well [16]. Power reduction due to RSOA chirping effect was examined theoretically and experimentally in OFDM $10 \mathrm{Gbps}$ long-range $(60 \mathrm{~km})$ transmission system by using commercially available RSOA of $<1.5 \mathrm{GHz}$ without any dispersion compensation [16]. To reduce the power loss a preliminary reinforcement of signal power was applied. This method allowed enhancing the sensitivity of the receiver by $5 \mathrm{~dB}$ (within the FEC BER).

\section{E. Optical Filtering Optimising}

Stricter AWG specifications and narrowband signals are necessary in case of high channel density to improve both the downstream and upstream transmissions. For this reason, the influence of MUX and DMUX filter bandwidth was investigated [5]. A theoretical wideband dynamic model was developed for numerical analysis of spatial and temporal distributions of photons and carriers in a bulk InP-InGaAsP RSOA. The influence of second order super-Gaussian optical filtering was estimated numerically for new generation 10 Gbps per channel DWDM system with RSOA, using NRZ signalling format with $25 \mathrm{GHz}$ channel spacing. It was revealed that the usage of lower extinction ratio of downstream signal provides an upstream and downstream signal transmission quality improvement without requiring strict filtering optimization.

\section{F. Data Rate Enhancing}

A modulation bandwidth for directly modulated colourless light sources depends on carrier lifetime in the active layer and typically is limited to $<3 \mathrm{GHz}[6,13]$. The most of WDM-PON realizations had demonstrated lower than $2.5 \mathrm{Gbps}$ transmission rate for this reason. Various methods were proposed for upstream transmission in 10 Gbps rate [2], [3], [6], [7], [13], [15]-[18]. One of them offered to use an OFDM which is also called the Discrete Multi-Tone (DMT) modulation [16]. The OFDM demonstrated a whole range of excellent features, i.e. robustness against fiber chromatic dispersion, data distribution flexibility for different subcarriers and high spectral efficiency. This method therefore is suitable to be used for the data rate increase with limited bandwidth and it can provide cost effective 10 Gbps WDM-PON systems design solutions [16]. OFDM or offline processing with the help of decision feedback equalization (DFE) and forward error correction (FEC) coding [3], [6] led to achieve $20 \mathrm{~km}$ transmission distance to certain limits of FEC. However, the BER achieved barely reached the FEC threshold even in back-to-back conditions for a 5 Gbps rate. $10 \mathrm{Gbps}$ or higher transmission rates are hardly achievable without equalization. To deal with this problem, there were proposed electronic and optical equalizers [11]. A cost-effective solution was based on duobinary modulation [3] and the adjustments of the RSOA's electro-optical (E/O) response to a $2.5 \mathrm{GHz}$ Bessel filter. The possibilities to remodulate continuous wave $(\mathrm{CW})$ and downstream signal by RSOA were investigated in [3]. An upstream of $85 \mathrm{~km}$ transmission at $10 \mathrm{Gbps}$ rate was achieved using standard single mode fiber and RSOA with $1.5 \mathrm{GHz}$ only. System performance was optimized only when applying detuned optical offset filtering and low-cost decision feedback equalization (DFE) at the OLT side, taking into account the RSOA chirp characteristics [3].

The using of additional optical components for higher transmission distances increased system costs. Therefore in order to force the carrier density to respond at a high speed (to increase LED modulation rate) it is appropriate to use instantaneous injection/depletion currents at the transition edges of the modulation signal. A 5 Gbps optical BPSK signal was obtained with modulation bandwidth $\sim 800 \mathrm{MHz}$ in the back-to-back condition [17]. Therefore there is no need to use the electronic equalization technique for RSOA limited modulation bandwidth compensation. Implementing it in WDM-PON digital coherent receiver a 10 Gbtps transmission at a wavelength in $80 \mathrm{~km}$ distance was reached. A wavelength distortion due to chirp-dispersion interaction was compensated in receiver using electronic dispersion compensator (EDC).

RSOA 10 Gbps operating rate was achieved by using optical delay interferometer (DI)) [2], [13]. DI operates as a two-tap optical equalizer as well as vestigial side-band filter. A 10 Gbps signal $20 \mathrm{~km}$ SSMF transmission was demonstrated in RSOA based WDM-PON with direct NRZ modulation [13].

In order to ensure a rate symmetry with downstream signal remodulation, the usage of a Differential Phase Shift Keying (DPSK) signal for downstream and on-off Keying $(\mathrm{OOK})$ signal remodulated for upstream with high extinction ratio in both directions were proposed [7]. Furthermore, these schemes required high injection power and sacrificed the extinction ratio (ER) of the downstream date to reduce the crosstalk to the upstream signal. DPSK uses downstream transmission which offers a number of advantages over conventional OOK modulation format [7]. For example, the optical phase modulator with one $\mathrm{LiNbO} 3$ crystal costs less than the Mach-Zehnder intensity modulator. In addition, DPSK modulation constant amplitude ensures a high ER and provides greater tolerance for the optical fiber nonlinearities and chromatic dispersion, thereby improving the system power budget (receiver sensitivity is increased by 3 dB) [7]. BER indicator revealed a good performance of the scheme presented as in the case of $\mathrm{CW}$ light insertion into RSOA, and optical spectrum of $30 \mathrm{~nm}$ enables 32 waves with $100 \mathrm{GHz}$ spacing. A $20 \mathrm{~km}$ distance was achieved in WDM-PON for $10 \mathrm{Gbps}$ and $5 \mathrm{Gbps}$ signals without 
dispersion compensation and significant power loss. The proposed scheme insures low transmission losses for both downstream and upstream and it is a practical solution for maintaining the desired rates and cost-effectiveness.

Even the possibility of $40 \mathrm{~Gb} / \mathrm{s}$ downstream using the Chirp Managed Directly Modulated Laser in WDM PON has been already demonstrated [2], but it still needs a high spectrum efficiency transmission scheme to increase the transmission distance limited by dispersion and to exploit different lengths between OLT and ONU. Fiber Bragg gratings (FBG) were analysed as a very attractive passive, linear, compact and tunable components because of their low costs, simple structure and easy installation and they are always used in dispersion compensation, optical filtering and optical equalization schemes. It was proposed to use the Remotely Pumped Erbium Doped Fiber Amplifier (RPEDFA) for $40 \mathrm{Gbps}$ downstream and $10 \mathrm{Gbps}$ upstream transmissions of long distance WDM-PON scheme with downstream transmission implemented using Quadrature Phase-Shift Keying (QPSK) transmitters and upstream transmission using FBG optical equalizer based on RSOA modulator and intensity DD receiver [2]. A number of BER measurements showed that not only does RPEDFA solve the issue of power budget for the long distance WDM-PON system, but also improves receiver sensitivity of upstream data.

100 Gbps WDM-PON can be realized using OFDM technique for high spectrum efficiency and resistance in various dispersions including the chromatic one [15], [18]. Since the OFDM requires high-speed electronics and complex optical circuits for multiplexing subcarriers, it may be difficult to realize a cost effective approach. Therefore, a solution was proposed to install $100 \mathrm{Gbps}$ system using coarse WDM (CWDM) [18], as is specified in 100 GBASELR4 standards. $100 \mathrm{GbE}$ signal was transmitted by four 25 Gbps CWDM channels. To reduce the costs, a RSOA was used as colourless light source instead of a high-speed external modulator. As it was already mentioned, the operation of the RSOA at gigabit rates is difficult to realize for a narrow bandwidth modulation bandwidth $(\mathrm{GHz})[6]$, [18]. For this reason RSOA mounted in butterfly packaging to reduce the electrical parasitics and increase the modulation bandwidth to $25.78 \mathrm{Gbps}$ [18]. Thus, RSOA modulation bandwidth was increased from $2.2 \mathrm{GHz}$ to $3.2 \mathrm{GHz}$ (40\%). Extremely limited RSOA bandwidth was compensated by using electronic equalization consisting of 17-tap FFE and 3-tap DFE and forward-error-correction (FEC). The following methods helped to achieve error-free $25.78 \mathrm{Gbps}$ transmission and $11 \mathrm{dBm}$ receiver sensitivity.

Various modulation formats were suggested to be used to solve this problem, i.e. Manchester coding [5] and subcarrier multiplexing (SCM) [13] rather than traditional NRZ format for downstream signal. In this case, modulation noise was outside the bandwidth of upstream signal and a noise of remodulation didn't have any influence on upstream signal BER characteristics. Thus it is possible to use high extinction ratio of logical 1 and 0 for downstream signal and to decrease power penalties due to remodulation.

\section{System With WaVElength Re-USe Performance SIMULATION}

This chapter presents the results of the research used to determine the following: if a signal sent from OLT could be used as a light source for ONU, what the length of transmission distance from ONU to OLT could be, what the value of additional attenuation could be on this transmission, how these characteristics depend on the data rate and the optical signal code used. The results obtained using OptiSystem software (support model is shown schematically in Fig. 2).

OLT transmitter consists of the following key components: data generator, the rate $R$ of which can be changed, pulse code generator that maintains NRZ and RZ coding, laser diode and external Mach-Zender modulator.

OLT optical transceiver has the ability to change logic 0 and logic 1 power levels of transmitted signal $\left(P_{0}\right.$ and $\left.P_{1}\right)$. OLT signal is transmitted to ONU inserting it through the optical circulator into the optical fiber which length $L$ can be changed and the next device is an optical attenuator, attenuation of which can be changed also. ONU signal is received through the coupler where it is split and routed to the receiver and RSOA. BER analyzer helps to evaluate the quality of the signal received in ONU (evaluation of $\mathrm{Q}$ factor). RSOA incoming signal is used as light source and RSOA reinforces it and modulates with ONU data that are generated by the signal generator and code generator.

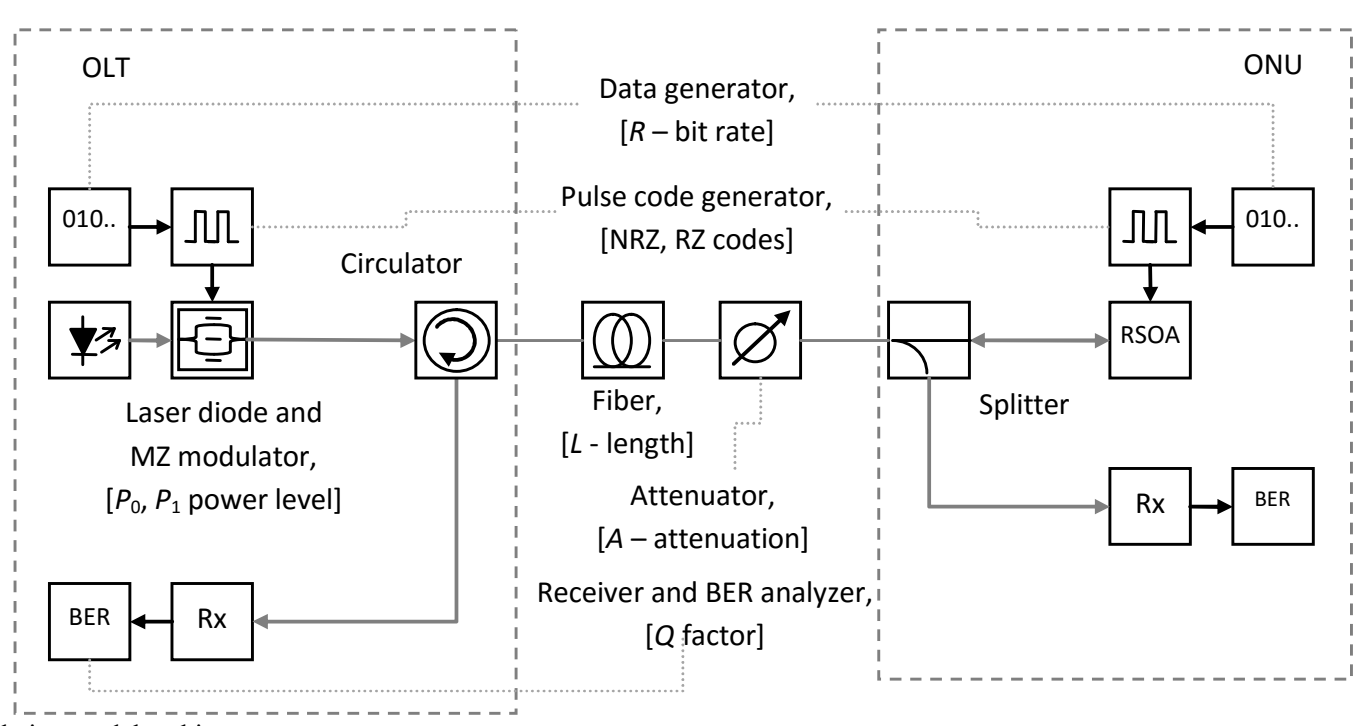

Fig. 2. The simulation model architecture. 
The parameters of RSOA are presented in Table I.

The upstream signal is transmitted from ONU through coupler, attenuator and fiber to OLT, where the circulator directs it to the receiver. The signal quality received in OLT is assessed by BER analyzer.The Fig. 3 shows the gain of RSOA as a function of wavelength.

TABLE I. THE PHYSICAL PARAMETERS OF RSOA.

\begin{tabular}{|c|c|c|c|}
\hline Name & Value & Name & Value \\
\hline $\begin{array}{c}\text { Input coupling } \\
\text { loss }\end{array}$ & $3 \mathrm{~dB}$ & $\begin{array}{c}\text { Optical } \\
\text { confinement } \\
\text { factor }\end{array}$ & 0.45 \\
\hline $\begin{array}{c}\text { Output } \\
\text { coupling loss }\end{array}$ & $3 \mathrm{~dB}$ & $\begin{array}{c}\text { Recombination } \\
\text { coefficient }\end{array}$ & $360 \cdot 10^{6} 1 / \mathrm{s}$ \\
\hline $\begin{array}{c}\text { Input facet } \\
\text { reflectivity }\end{array}$ & $50 \cdot 10^{-6}$ & $\begin{array}{c}\text { Recombination } \\
\text { coefficient }\end{array}$ & $\begin{array}{c}0.56 \cdot 10^{-15} \\
\mathrm{~m}^{3} / \mathrm{s}\end{array}$ \\
\hline $\begin{array}{c}\text { Output facet } \\
\text { reflectivity }\end{array}$ & 0.99 & $\begin{array}{c}\text { Recombination } \\
\text { coefficient }\end{array}$ & $30 \cdot 10^{-42} \mathrm{~m}^{6} / \mathrm{s}$ \\
\hline Active length & $6 \cdot 10^{-4} \mathrm{~m}$ & Group velocity & $75 \cdot 10^{6} \mathrm{~m} / \mathrm{s}$ \\
\hline Tapper length & $1 \cdot 10^{-4} \mathrm{~m}$ & Temperature & $300 \mathrm{~K}$ \\
\hline Active area & $0.16 \cdot 10^{-12} \mathrm{~m}^{2}$ & $\begin{array}{c}\text { Junction ideality } \\
\text { factor }\end{array}$ & 2 \\
\hline
\end{tabular}

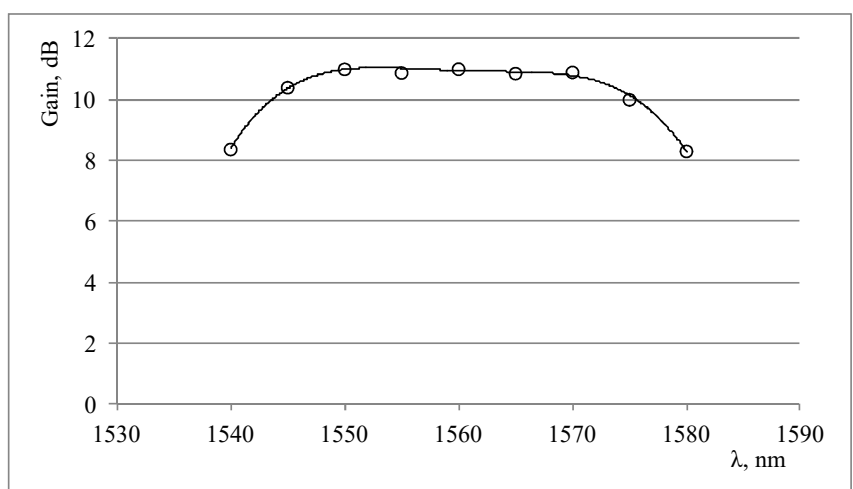

Fig. 3. The gain of RSOA as a function of wavelength.

For further simulation results the $1554.52 \mathrm{~nm}$ (193.1 THz) wavelength was used.

A transmission quality of digital optical signal is expressed by Q factor which can be converted to BER. For example, the Gigabit Ethernet standard requires that the $\mathrm{BER} \leq 10^{-12}$, which corresponds to $Q \geq 7$.

\section{A. Signal Level Impact Assessing}

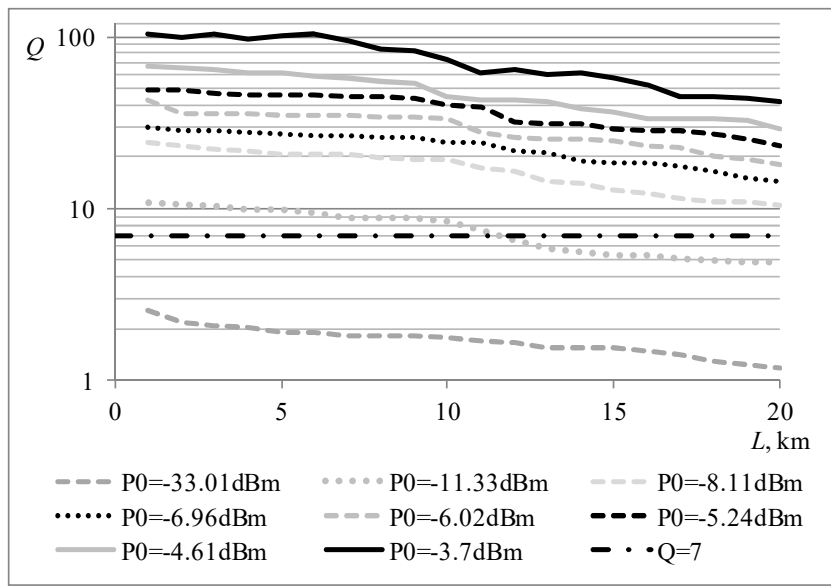

Fig. 4. $Q$ factor of OLT received signal as a function of $L$ and $P_{0}$, given $P_{1}=-3.01 \mathrm{dBm}, R=1.25 \mathrm{Gbps}, A=0 \mathrm{~dB}$, OLT transmitter uses external modulation with NRZ code, ONU transmitter uses RSOA with NRZ code.

In this case, RSOA uses the signal received from OLT as a light source. It is understandable that if the OLT does not send anything or logic 0 signal level is too low, RSOA in ONU will not be able to remodulate the signal and send it to OLT. It is therefore appropriate to determine what the level of logic $0\left(P_{0}\right)$ for signal continuously transmitted from the OLT should be.

The length of optical fiber and the level of logical $0\left(P_{0}\right)$ were changed during this investigation, when the logical level $1\left(P_{1}\right)$ was left constant $(-3.01 \mathrm{dBm})$, additional constant attenuation was $0 \mathrm{~dB}$, and OLT and ONU used NRZ coding technique.

The results of this investigation are displayed in Fig. 4, when data is transmitted at the rate of $R=1.25 \mathrm{Gbps}$ (based on Gigabit Ethernet or GPON upload rate in physical level).

The simulation results were obtained changing transmission rate to $R=2.5 \mathrm{Gbps}$ (based on GPON download rate in physical level) (Fig. 5).

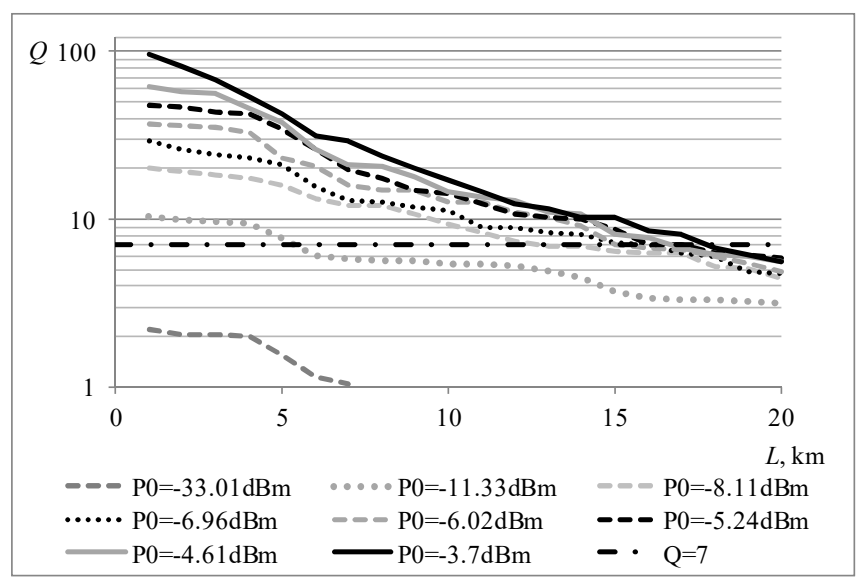

Fig. 5. $Q$ factor of OLT received signal as a function of $L$ and $P_{0}$, given $P_{1}=-3.01 \mathrm{dBm}, R=2.5 \mathrm{Gbps}, A=0 \mathrm{~dB}$, OLT transmitter uses external modulation with NRZ code, ONU transmitter uses RSOA with NRZ code.

These results revealed (Fig. 5) that the distance of $18 \mathrm{~km}$ was the maximum distance which still allowed to transmit a 2.5 Gbps signal from ONU to OLT with quality level of $Q \geq$ 7, when $P_{0}=-3.7 \mathrm{dBm}$.

\section{B. Determination of Additional Attenuation between OLT and $O N U$}

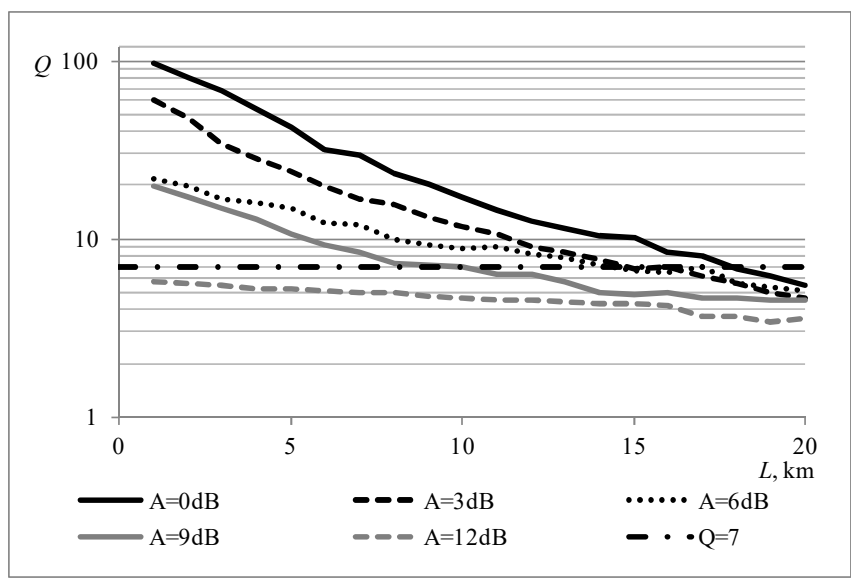

Fig. 6. $Q$ factor of OLT received signal as a function of $L$ and $A$, given $P_{0}=$ $-3.7 \mathrm{dBm}, P_{1}=-3.01 \mathrm{dBm}, R=2.5 \mathrm{Gbps}$, OLT transmitter uses external modulation with NRZ code, ONU transmitter uses RSOA with NRZ code.

An attenuation of signal transmitted in RSOA-based WDM-PON access can be evaluated by changing 
attenuation of attenuator (parameter $A$ ). A dynamic of $Q$ was revealed when increasing attenuation $A$ and optical fiber length $L$. The results are presented in Fig. 6.

When $A=12 \mathrm{~dB}$, then $Q<7$ for any fiber length, regardless of the transmission rate $R$.

\section{Determination of Code Influence}

A simulation model allows to assess the impact of OLT and ONU coding techniques on the signal transmitting quality. For this purpose, a series of simulations were carried out with different OLT and ONU coding generators setting the maximum allowable attenuation value to $A=$ $9 \mathrm{~dB}$.

The results obtained with transmission rate $R=2.5 \mathrm{Gbps}$ are presented in Fig. 7.

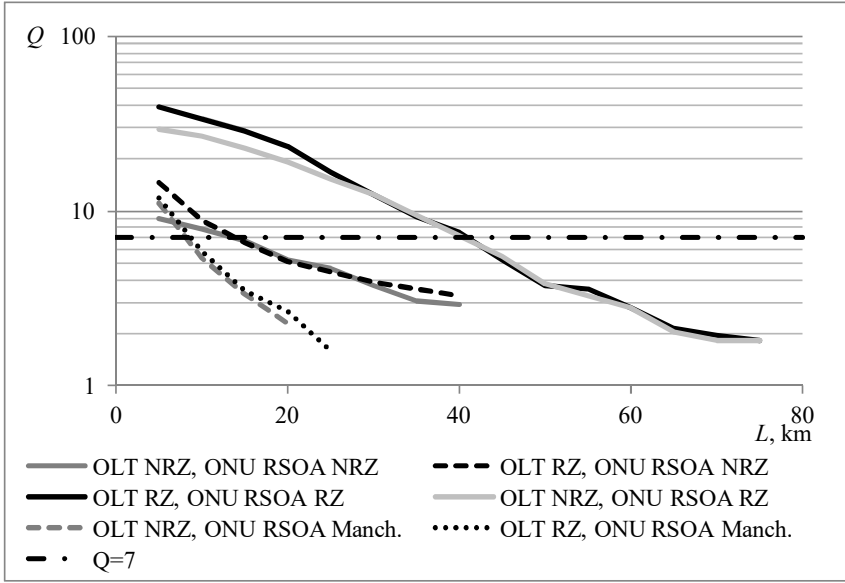

Fig. 7. Q factor of OLT received signal as a function of $\mathrm{L}$ and code type, given $\mathrm{P} 0=-3.7 \mathrm{dBm}, \mathrm{P} 1=-3.01 \mathrm{dBm}, \mathrm{A}=9 \mathrm{~dB}, \mathrm{R}=2.5 \mathrm{Gbps}$, OLT transmitter uses external modulation, ONU transmitter uses RSOA.

They revealed that it is better to use RZ coding since optical signal from RSOA can be transmitted in a much longer distance. It is possible to explain this by the fact that synchronization between symbols is better supported using RZ coding allowing good separation of symbols obtained in RSOA remodulation.

The usage of Manchester code for RSOA optical signal coding does not give better results, because, in case of Manchester code one data bit is coded by two optical signal bits.

\section{CONCLUSIONS}

A comprehensive problem analysis of RSOA-based WDM-PON revealed many factors affecting system performance. The most important of them are various optical signal reflections, in-band crosstalk, nonlinearity, RSOA chirp, resulting in optical signal power penalties as well as optical filtering problems at high WDM channels density. For these reasons, a number of investigations were carried out optimising the performance of that system.

A power level of downstream signal from OLT which is received in ONU and remodulated with RSOA is very important. Approaching (increasing) a power level of logic 0 to logic 1 of downstream signal allows to improve a quality of RSOA remodulation or increase possible transmission distance. Although remodulation noise is reduced when reducing the logical 1 and 0 extinction ratio of downstream signal, this may affect the sensitivity of downstream signal receiver. Therefore, it is appropriate to introduce a compromise ratio between downstream and upstream signal characteristic, i.e. it is appropriate to determine an optimal difference between power levels of logical 0 and 1 for both transmittion directions: from ONU to OLT and in opposite one. In further research the optimization of power levels of logical 0 and 1 and signal power splitting between ONU's RSOA and receiver should be done.

It was found that RZ coding was better suited in RSOA remodulation; for example, it allowed achieving $1.25 \mathrm{Gbps}$ signal at 8 times longer transmission distance than using NRZ coding. Hence, further research would be needed to assess the influence on transmittion distance for upstream signal remodulated in RSOA using another modulation and coding techniques in OLT node.

\section{REFERENCES}

[1] P. J. Urban, A. M. J. Koonen, G. D. Khoe, H. de Waardt "Interferometric crosstalk reduction in an RSOA-Based WDM passive optical network", Journal of Lightwave Technology, vol. 27, no. 22, pp. 4943-4953, 2009. [Online]. Available: http://dx.doi.org/ 10.1109/JLT.2009.2026916

[2] Z. Zhang, X. Chen, L. Wang, M. Zhang, “40-Gb/s QPSK downstream and $10-\mathrm{Gb} / \mathrm{s}$ RSOA based upstream transmission in long-reach WDM PON employing remotely pumped EDFA and FBG optical equalizer", in 8th Int. Conf. on Communications and Networking, China, 2013, pp. 788-791. [Online]. Available: http://dx.doi.org/10.1109/ chinacom.2013.6694702

[3] I. Papagiannakis, M. Omella, D. Klonidis, J. A. Lazaro Villa, A. N. Birbas, J. Kikidis, I. Tomkos, J. Prat, "Design characteristics for a full-duplex IM/IM bidirectional transmission at $10 \mathrm{~Gb} / \mathrm{s}$ using low bandwidth RSOA", Journal of Lightwave Technology, vol. 28, no. 7, pp. 1094-1101, 2010. [Online]. Available: http://dx.doi.org/10.1109/ JLT.2009.2039195

[4] Y. J. Lee, K. Y. Cho, A. Murakami, A. Agata, Y. Takushima, Y. C. Chung, "Reflection tolerance of RSOA-based WDM PON", in Optical Fiber communication/National Fiber Optic Engineers Conf., San Diego, CA, 2008, pp.1-3. [Online]. Available: http://dx.doi.org/10.1109/ofc.2008.4528609

[5] Z. Vujicic, N. B. Pavlovic, A. Teixeir, "Optical filtering optimization for NRZ coding format in RSOA-Based DWDM PON", in Transparent 14th Int. Conf. Optical Networks, 2012, pp. 1-4. [Online]. Available: http://dx.doi.org/10.1109/icton.2012.6253806

[6] K. Y. Cho, Y. Takushima, Y. C. Chung, "10-Gb/s operation of RSOA for WDM PON", IEEE Photonics Technology Letters, vol. 20, no. 18, pp. $1533-1535,2008$. [Online]. Available: http://dx.doi.org/10. 1109/LPT.2008.928834

[7] J. Zhang, X. Yuan, Y. Gu, Y. Huang, M. Zhang, Y. Zhang, “A novel bidirectional RSOA based WDM-PON with downstream DPSK and upstream re-modulated OOK data", in 11th Int. Conf. Transparent Optical Network, Azores, 2009, pp. 1-5.

[8] K. Y. Cho, Y. J. Lee, H. Y. Choi, A. Murakami, A. Agata, Y. Takushima, Y. C. Chung, "Effects of reflection in RSOA-based WDM PON utilizing remodulation technique", Journal of Lightwave Technology, vol. 27, no. 10, pp. 1286-1295, 2009. [Online]. Available: http://dx.doi.org/10.1109/JLT.2009.2016580

[9] K. Y. Cho, Y. Takushima, Y. C. Chung, "10-Gb/s operation of RSOA for WDM PON", IEEE Photon. Technol. Letters, vol. 20, no. 18, pp. 1533-1535, 2008. [Online]. Available: http://dx.doi.org/10.1109/ LPT.2008.928834

[10] R. Poboril, P. Siska, J. Latal, M. Smrz, J. Cubik, S. Kepak, V. Vasinek, "Measuring optimal length of the amplifying fiber in different working conditions of the amplifier", Advances in Electrical and Electronic Engineering (AEEE 2014), vol. 12, no. 6, pp. 557566, 2014.

[11] Ch. H. Kim, "Dependence of in-band incoherent crosstalk-induced penalty on seed source in RSOA-based WDM-PONs", Photonics Technology Letters, vol. 24, no. 7, pp. 581-583, 2012. [Online]. Available: http://dx.doi.org/10.1109/LPT.2012.2183864

[12] G. P. Agrawal, N. A. Olson, "Self-phase modulation and spectral broadening of optical pulses in semiconductor laser amplifiers", IEEE J. Quant. Electron., vol. 25, no. 11, pp. 2297-2306, 1989. [Online]. Available: http://dx.doi.org/10.1109/3.42059 
[13] H. Kim, "10-Gbps upstream transmission for WDM-PON using RSOA and delay interferometer", in the National Fiber Optic Engineers Conf., 2011, pp. 1-3. [Online]. Available: http://dx.doi.org/ 10.1364/ofc.2011.omp8

[14] W.-S. Jang, H.-C. Kwon, S.-K. Han, "Suppression of Rayleigh backscattering in a bidirectional WDM optical link using clipped direct modulation", in IEEE Proc.-Optoelectron., vol. 151, no. 4, 2004, pp. 219-222. [Online]. Available: http://dx.doi.org/10.1049/ipopt:20040782

[15] X. Guo, R. Lin, Y.Tan, S. Zhou, J. Zhan, "Analysis of nonlinear effects in RSOA-based OFDM-PON", in Proc. SPIE-OSA-IEEE Asia Communications and Photonics Conf. and Exibition, Shanghai, China, 2011, pp. 1254-1259.

[16] P. Choudhury, M. Presi, G. Cossu, A. Chiuchiarelli, E. Ciaramella, "Chirped RSOA modulation by using adaptive OFDM for long reach
WDM-PONs", in Int. Conf. on Photonics in Switching (PS), 2012, pp. $1-3$.

[17] A. Agata, T. Sano, K. Y. Cho, U. H. Hong, K. Nishimura, H. Tanaka, M. Suzuki, Y. C. Chung, "Upstream transmission of $10-\mathrm{Gb} / \mathrm{s}$ QPSK signal generated by directly modulating RSOA with instantaneous injection/depletion currents in coherent WDM PON", in Optical Fiber Communication Conf. and Exposition 2012, and the National Fiber Optic Engineers Conf. (OFC/NFOEC 2012), Los Angeles, CA, 2012, pp. 1-3. [Online]. Available: http://dx.doi.org/10.1364/ofc.2012. oth1f.1

[18] K. Y. Cho, B. S. Choi, Y. Takushima, Y. C. Chung, "25.78-Gb/s operation of RSOA for next-generation optical access networks", IEEE Photonics Technology Letters, vol. 23, no. 8, pp. 495-497, 2011. [Online]. Available: http://dx.doi.org/10.1109/LPT 2011.2112759 